\title{
Fast variance predictions for 3D cone-beam CT with quadratic regularization
}

\author{
Yingying Zhang-O'Connor and Jeffrey A. Fessler ${ }^{a}$ \\ ${ }^{a}$ Department of Electrical Engineering and Computer Science, The University of Michigan, 1301 \\ Beal Avenue, Ann Arbor, USA
}

\begin{abstract}
Fast and accurate variance/covariance predictions are useful for analyzing the statistical characteristics of the reconstructed images and may aid regularization parameters selection. The existing methods, the matrix-based method and its DFT approximations, are impractical for realistic data size in X-ray CT. We have previously addressed this problem in $2 \mathrm{D}$ fan-beam CT by proposing "analytical" approaches, the simplest of which requires computation equivalent to one backprojection and some summations. This paper extends these approaches to 3D step-and-shoot "cylindrical" cone-beam CT.
\end{abstract}

Keywords: variance approximation, local discrete Fourier analysis, cone-beam tomography, penalized-likelihood image reconstruction

\section{INTRODUCTION}

Statistical reconstruction methods are usually nonlinear and shift-variant. To analyze the statistical characteristics of the reconstructed images from these methods, one would often like to predict the variance of pixel/voxel values. The variance information provides an uncertainty measure of the reconstructed image and can also be very useful for regularization parameter selection.

The existing prediction methods derived in ${ }^{1}$ for penalized likelihood estimators are computationally expensive and only practical when the variances prediction are needed at a few image locations.

As shown in, ${ }^{1}$ the $p \times p$ covariance matrix for quadratically penalized likelihood estimators is approximately

$$
\boldsymbol{K} \triangleq \operatorname{Cov}\{\hat{\boldsymbol{\mu}}\} \approx\left(\boldsymbol{A}^{\prime} \boldsymbol{W} \boldsymbol{A}+\alpha \boldsymbol{R}\right)^{-1} \boldsymbol{A}^{\prime} \boldsymbol{W} \boldsymbol{A}\left(\boldsymbol{A}^{\prime} \boldsymbol{W} \boldsymbol{A}+\alpha \boldsymbol{R}\right)^{-1},
$$

where $\boldsymbol{\mu}=\left[\mu_{1}, \ldots, \mu_{p}\right]^{\prime}, \boldsymbol{A}$ is the system matrix, $\boldsymbol{Y}=\left[y_{1}, \ldots, y_{n}\right]^{\prime}$ denotes the noisy measurements, $\boldsymbol{R}$ is the Hessian matrix of the roughness penalty and $\alpha$ is the regularization parameter controlling the noise and resolution tradeoff.

In the spirit of the local shift-invariance approximations, ${ }^{5}$ we approximate the covariance matrix in (1) near a given location $\vec{n}_{0}$ by

$$
\breve{\boldsymbol{K}}_{0} \triangleq\left(\mathrm{F}_{0}+\alpha \boldsymbol{R}_{0}\right)^{-1} \mathrm{~F}_{0}\left(\mathrm{~F}_{0}+\alpha \boldsymbol{R}_{0}\right)^{-1},
$$

where $\mathrm{F}_{0}$ and $\boldsymbol{R}_{0}$ are the $\left(N_{1} N_{2} N_{3}\right) \times\left(N_{1} N_{2} N_{3}\right)$ block Toeplitz with Toeplitz blocks (BTTB) approximations corresponding to $\boldsymbol{A}^{\prime} \boldsymbol{W} \boldsymbol{A}$ and $\boldsymbol{R}$, respectively. In practical computation of predicted variances at a few image locations, circulant approximations and DFTs are usually used as follows: ${ }^{2,3}$

$$
\operatorname{Var}\{\hat{\mu}[\vec{n}]\} \approx \frac{1}{N_{1} N_{2} N_{3}} \sum_{\vec{k}=\overrightarrow{0}}^{\vec{N}-1} P_{d 0}[\vec{k}],
$$

where $\vec{N}=\left(N_{1}, N_{2}, N_{3}\right)$,

$$
P_{d 0}[\vec{k}] \triangleq \frac{\Gamma_{0}[\vec{k}]}{\left(\Gamma_{0}[\vec{k}]+\alpha \Omega_{0}[\vec{k}]\right)^{2}},
$$

Further author information: (Send correspondence to Yingying Zhang-O'Connor) Yingying Zhang-O’Connor: E-mail: yzz@eecs.umich.edu

Medical Imaging 2007: Physics of Medical Imaging, edited by Jiang Hsieh, Michael J. Flynn, Proc. of SPIE Vol. 6510, 65105W, (2007) - 1605-7422/07/\$18 - doi: 10.1117/12.710312 
with $\mathrm{F}_{0} \approx \boldsymbol{Q} \boldsymbol{\Gamma}_{0} \boldsymbol{Q}^{\prime}, \quad \boldsymbol{R}_{0} \approx \boldsymbol{Q} \boldsymbol{\Omega}_{0} \boldsymbol{Q}^{\prime}$ where $\boldsymbol{Q}$ is the 3D $\left(N_{1}, N_{2}, N_{3}\right)$-point orthonormal DFT matrix, and $\boldsymbol{\Gamma}_{0}$ and $\boldsymbol{\Omega}_{0}$ are diagonal matrices with diagonal elements $\Gamma_{0}[\vec{k}]$ and $\Omega_{0}[\vec{k}]$ that are the 3D DFT coefficients of the local impulse response of $\boldsymbol{A}^{\prime} \boldsymbol{W} \boldsymbol{A}$ and $\boldsymbol{R}$ near $\vec{n}_{0}$, respectively. Computing this DFT approximation is still expensive for realistic image sizes when the variance is computed for all or many pixels, particularly for shift-variant systems like fan-beam CT and cone-beam CT.

To aid regularization parameters' selection, we would like to obtain the variance information for all or many image locations. Therefore, a new fast and accurate prediction method is needed.

\section{GENERAL VARIANCE PREDICTION FOR 3D TOMOGRAPHY}

We have proposed new "analytical" approaches to predict the approximate variance maps of 2D images that are reconstructed by penalized-likelihood estimation with quadratic regularization in fan-beam geometries. ${ }^{4,5}$ In this paper, we expand these approaches to 3D step-and-shoot cone-beam CT by applying the same principles: "local shift invariance" approximation and "local Fourier analysis".

We approximate (2) by the 3D discrete-space Fourier transform (DSFT) as follows:

$$
\operatorname{Var}\{\hat{\mu}[\vec{n}]\} \approx \int_{-\pi}^{\pi} \int_{-\pi}^{\pi} \int_{-\pi}^{\pi} P_{d 0}(\vec{\omega}) \frac{\mathrm{d} \vec{\omega}}{(2 \pi)^{3}}
$$

where $P_{d 0}(\vec{\omega})$ is the local spectrum of the covariance matrix, given as follows:

$$
P_{d 0}(\vec{\omega}) \triangleq \frac{H_{\mathrm{d} 0}(\vec{\omega})}{\left[H_{\mathrm{d} 0}(\vec{\omega})+\alpha R_{\mathrm{d} 0}(\vec{\omega})\right]^{2}},
$$

where $H_{\mathrm{d} 0}(\vec{\omega})$ is the local frequency response of the Gram matrix $\boldsymbol{A}^{\prime} \boldsymbol{W} \boldsymbol{A}$ and $R_{\mathrm{d} 0}(\vec{\omega})$ is the local frequency response of $\boldsymbol{R}$ near $\vec{n}_{0}$.

Let $\vec{\Delta}=\left(\Delta_{\mathrm{X}}, \Delta_{\mathrm{Y}}, \Delta_{\mathrm{Z}}\right)$ denote the sample spacings in the reconstructed image. Make the change of variable, $\vec{\omega}=$ $(2 \pi \varrho) \vec{\Delta} \odot \vec{e}_{\Phi, \Theta}$ where $\vec{e}_{\Phi, \Theta}=(\cos \Phi \cos \Theta, \sin \Phi \cos \Theta, \sin \Theta)$, and $\odot$ denotes element-by-element multiplication. We rewrite (3) in terms of spherical frequency coordinates $(\varrho, \Phi, \Theta)$ as follows:

$$
\operatorname{Var}\{\hat{\mu}[\vec{n}]\} \approx \Delta_{\mathrm{X}} \Delta_{\mathrm{Y}} \Delta_{\mathrm{Z}} \int_{-\pi}^{\pi} \int_{0}^{2 \pi} \int_{0}^{\varrho_{\max }(\Phi, \Theta)} P_{0}(\varrho, \Phi, \Theta) \varrho^{2}|\cos \Theta| \mathrm{d} \varrho \mathrm{d} \Phi \mathrm{d} \Theta
$$

where $\varrho_{\max }(\Phi, \Theta)=1 /\left\{2 \min \left(\frac{\Delta_{\mathrm{X}}}{|\cos \Phi \cos \Theta|}, \frac{\Delta_{\mathrm{Y}}}{|\sin \Phi \cos \Theta|}, \frac{\Delta_{\mathrm{Z}}}{|\sin \Theta|}\right)\right\}$, and we define

$$
P_{0}(\varrho, \Phi, \Theta) \triangleq P_{\mathrm{d} 0}\left(2 \pi \varrho \vec{\Delta} \odot \vec{e}_{\Phi, \Theta}\right)=\frac{H_{0}(\varrho, \Phi, \Theta)}{\left[H_{0}(\varrho, \Phi, \Theta)+\alpha R_{0}(\varrho, \Phi, \Theta)\right]^{2}} .
$$

We define $H_{0}$ and $R_{0}$ similarly in terms of $H_{\mathrm{d} 0}$ and $R_{\mathrm{d} 0}$. If we find analytical expressions for $H_{0}$ and $R_{0}$, then the approximation (5) can lead to faster alternatives to the DFT approach (2). The analytical variance prediction in (5) is applicable to any 3D CT geometry. We focus on step-and-shoot 3D cone-beam CT here. To use (5), we need to find $H_{0}(\varrho, \Phi, \Theta)$ and $R_{0}(\varrho, \Phi, \Theta)$ first.

\section{STEP-AND-SHOOT CONE-BEAM GEOMETRY}

Consider an ideal "cylindrical" step-and-shoot cone-beam tomography: the source can be at any point on a cylinder of radius $D_{\mathrm{s} 0}$ centered along the z-axis. The source position $\vec{p}_{0}$ can be parameterized by two variables $(\beta, \zeta)$ as follows:

$$
\vec{p}_{0}=\left(\begin{array}{c}
-D_{\mathrm{s} 0} \sin \beta \\
D_{\mathrm{s} 0} \cos \beta \\
\zeta
\end{array}\right),
$$

where $D_{\mathrm{s} 0}$ is the source to rotation center distance, $\beta$ is the angle of the source relative to the $y$ axis, and $\zeta$ is the z-axis position of source. 
We focus on the 2D cylindrical detector that moves with the cone vertex here. We introduce local/relative coordinates $(s, t)$ on the detector plane, where $s$ is the arc length along each row, and the t-axis is parallel to the z-axis. A point on the $2 \mathrm{D}$ detector can be expressed as

$$
\vec{p}_{1}=\left(\begin{array}{c}
D_{\mathrm{sd}} \sin \gamma \cos \beta+\left(D_{\mathrm{sd}} \cos \gamma-D_{\mathrm{s} 0}\right) \sin \beta \\
D_{\mathrm{sd}} \sin \gamma \sin \beta-\left(D_{\mathrm{sd}} \cos \gamma-D_{\mathrm{s} 0}\right) \cos \beta \\
t+\zeta
\end{array}\right)=\left(\begin{array}{c}
D_{\mathrm{sd}} \sin \varphi \\
-D_{\mathrm{sd}} \cos \varphi \\
t
\end{array}\right)+\vec{p}_{0},
$$

where $D_{0 \mathrm{~d}}=D_{\mathrm{sd}}-D_{\mathrm{s} 0}$ is the isocenter to detector distance and

$$
\gamma=\gamma(s) \triangleq \frac{s}{D_{\mathrm{sd}}}, \quad \varphi=\varphi(s, \beta) \triangleq \gamma(s)+\beta .
$$

The direction vector of a ray from $\vec{p}_{0}$ to $\vec{p}_{1}$ can then be expressed as

$$
\vec{e}_{3}(\vec{\gamma})=\frac{\vec{p}_{1}-\vec{p}_{0}}{\left\|\vec{p}_{1}-\vec{p}_{0}\right\|}=\frac{1}{\sqrt{t^{2}+D_{\mathrm{sd}}^{2}}}\left(\begin{array}{c}
D_{\mathrm{sd}} \sin \varphi \\
-D_{\mathrm{sd}} \cos \varphi \\
t
\end{array}\right)=\left(\begin{array}{c}
\cos \theta \sin \varphi \\
-\cos \theta \cos \varphi \\
\sin \theta
\end{array}\right),
$$

where $\vec{\gamma}=(\varphi, \theta)$ and

$$
\theta=\theta(t) \triangleq \arctan \left(\frac{t}{D_{\mathrm{sd}}}\right)
$$

The projection plane is perpendicular to $\vec{e}_{3}(\vec{\gamma})$, specified by

$$
\vec{e}_{1}(\vec{\gamma})=(\cos \varphi, \sin \varphi, 0), \quad \vec{e}_{2}(\vec{\gamma})=(-\sin \varphi \sin \theta, \cos \varphi \sin \theta, \cos \theta) .
$$

The corresponding Cartesian coordinates $(u, v)$ can be found by

$$
\begin{aligned}
u(s) & =\vec{p}_{1} \cdot \vec{e}_{1}(\vec{\gamma})=D_{\mathrm{sd}} \sin \gamma \cos \gamma-\left(D_{\mathrm{sd}} \cos \gamma-D_{\mathrm{s} 0}\right) \sin \gamma=D_{\mathrm{s} 0} \sin \gamma(s) \\
v(s, t, \zeta) & =\vec{p}_{1} \cdot \vec{e}_{2}(\vec{\gamma})=D_{\mathrm{s} 0} \cos \gamma(s) \sin \theta(t)+\zeta \cos \theta(t),
\end{aligned}
$$

since $-D_{\mathrm{sd}} \sin \theta(t)+t \cos \theta(t)=0$. Combining (9), (11), (12) and (13), we have the cone-to-parallel rebinning relations.

\subsection{Local Impulse Response of Gram Matrix}

To predict variance images in fan-beam transmission tomography we need to determine the local frequency response $H_{0}(\rho, \Phi, \Theta)$, or equivalently $H_{\mathrm{d} 0}(\vec{\omega})$. We first find the local impulse response.

Consider the 3D object model based on a common basis function $\chi(\vec{x})$ superimposed on a $N \times M \times L$ Cartesian grid as follows:

$$
\mu(\vec{x})=\sum_{\vec{n} \in \mathcal{S}} \mu[\vec{n}] \chi\left(\frac{1}{\vec{\Delta}} \odot\left(\vec{x}-\vec{x}_{c}[\vec{n}]\right)\right),
$$

where $\mathcal{S} \triangleq\left\{\vec{n}_{j}: j=1, \ldots, p\right\}$ denotes the subset of the $N \times M \times L$ lattice that is estimated and $\vec{x}_{c}[\vec{n}]$ denotes the center of the $\vec{n}$ th basis function. The grid spacing is $\vec{\Delta}=\left(\Delta_{\mathrm{X}}, \Delta_{\mathrm{Y}}, \Delta_{\mathrm{Z}}\right)$. We consider the case $\Delta_{\mathrm{X}}=\Delta_{\mathrm{Y}}$ hereafter, but we allow $\Delta_{\mathrm{X}} \neq \Delta_{\mathrm{z}}$. Typically $\vec{w}_{\vec{x}}=(\vec{N}-1) / 2+\vec{c}_{\vec{x}}, \quad \vec{x}_{c}[\vec{n}]=\left(\vec{n}-\vec{w}_{\vec{x}}\right) \odot \vec{\Delta}, \quad \vec{n} \in \mathcal{S}$, where the user-selectable parameter $\vec{c}_{\vec{x}}$ denotes an optional spatial offset for the object center.

Assume that the detector blur $b(s, t)$ is shift invariant, independent of source position $(\beta, \zeta)$ and acts only along the $s$ and $t$ coordinates. (This could be generalized to the case of locally shift-invariant blur.) Then we model the mean projections as follows:

$$
\bar{y}_{\varphi}\left[s_{k}, t_{\ell} ; \beta, \zeta\right]=\iint b\left(s_{k}-s^{\prime}, t_{\ell}-t^{\prime}\right) p\left(\tilde{u}\left(s^{\prime}\right), \tilde{v}\left(s^{\prime}, t^{\prime}, \zeta\right) ; \tilde{\varphi}\left(s^{\prime}, \beta\right), \tilde{\theta}\left(t^{\prime}\right)\right) \mathrm{d} s^{\prime} \mathrm{d} t^{\prime}
$$

for $s_{k}=\left(k-w_{\mathrm{S}}\right) \Delta_{\mathrm{S}}, \quad t_{\ell}=\left(\ell-w_{t}\right) \Delta_{t}$ and $k=0, \ldots, n_{s}-1 ; \ell=0, \ldots, n_{t}-1$, where $\Delta_{\mathrm{S}}$ is the sample spacing in $s$, $\Delta_{t}$ is the sample spacing in $t, w_{\mathrm{S}}$ and $w_{t}$ are defined akin to $\vec{w}_{\vec{x}}$, and $p(u, v ; \varphi, \theta)$ is the line integral of $\mu(\vec{x})$. 
Let $g(u, v ; \varphi, \theta)$ denote the Radon transform of $\chi(\vec{x})$ at angle $(\varphi, \theta)$. By shifting and scaling properties of Radon transform, we have

$$
\chi\left(\frac{1}{\vec{\Delta}} \odot\left(\vec{x}-\vec{x}_{c}[\vec{n}]\right)\right) \stackrel{3 \mathrm{D} \text { Xray }}{\longleftrightarrow} \frac{\Delta_{\mathrm{X}} \Delta_{\mathrm{Z}}}{c(\theta)} g\left(\frac{u-u_{\varphi}[\vec{n}]}{\Delta_{\mathrm{X}}}, \frac{v-v_{\varphi, \theta}[\vec{n}]}{c(\theta)} ; \varphi, \arctan \left(\frac{\Delta_{\mathrm{X}}}{\Delta_{\mathrm{Z}}} \tan \theta\right)\right)
$$

where $c(\theta) \triangleq \sqrt{\Delta_{\mathrm{X}}^{2} \sin ^{2} \theta+\Delta_{\mathrm{Z}}^{2} \cos ^{2} \theta}$, and $u_{\varphi}[\vec{n}] \triangleq \vec{x}_{c}[\vec{n}] \cdot \vec{e}_{1}(\varphi), \quad v_{\varphi, \theta}[\vec{n}] \triangleq \vec{x}_{c}[\vec{n}] \cdot \vec{e}_{2}(\varphi, \theta)$.

Substituting the basis expansion model in (14) for the object into the measurement model (15) and simplfying leads to the linear model $\bar{y}_{\varphi}\left[s_{k}, t_{\ell} ; \beta, \zeta\right]=\sum_{\vec{n} \in \mathcal{S}} a\left(s_{k}, t_{\ell} ; \beta, \zeta ; \vec{n}\right) \mu[\vec{n}]$, where the cone-beam system matrix elements are samples of the following cone-beam projection of a single basis function centered at $\vec{x}_{c}[\vec{n}]$ :

$$
\begin{aligned}
& a(s, t ; \beta, \zeta ; \vec{n})=\iint b\left(s-s^{\prime}, t-t^{\prime}\right) \frac{\Delta_{\mathrm{X}} \Delta_{\mathrm{Z}}}{c\left(\tilde{\theta}\left(t^{\prime}\right)\right)} \\
& \cdot g\left(\frac{\tilde{u}\left(s^{\prime}\right)-u_{\tilde{\varphi}\left(s^{\prime}, \beta\right)}[\vec{n}]}{\Delta_{\mathrm{X}}}, \frac{\tilde{v}\left(s^{\prime}, t^{\prime}, \zeta\right)-v_{\tilde{\varphi}\left(s^{\prime}, \beta\right), \tilde{\theta}\left(t^{\prime}\right)}[\vec{n}]}{c\left(\tilde{\theta}\left(t^{\prime}\right)\right)} ; \tilde{\varphi}\left(s^{\prime}, \beta\right), \tilde{\theta}\left(\frac{\Delta_{\mathrm{X}}}{\Delta_{\mathrm{Z}}} t^{\prime}\right)\right) \mathrm{d} s^{\prime} \mathrm{d} t^{\prime},
\end{aligned}
$$

where

$$
\arctan \left(\frac{\Delta_{\mathrm{x}}}{\Delta_{\mathrm{z}}} \tan \tilde{\theta}\left(t^{\prime}\right)\right)=\arctan \left(\frac{\Delta_{\mathrm{x}}}{\Delta_{\mathrm{z}}} \frac{t^{\prime}}{D_{\mathrm{sd}}}\right)=\tilde{\theta}\left(\frac{\Delta_{\mathrm{x}}}{\Delta_{\mathrm{z}}} t^{\prime}\right)
$$

To proceed, we rebin (16) into parallel coordinates. Reparameterizing variables $s^{\prime}$ and $t^{\prime}$ according to the inversion of cone-to-parallel rebinning as follows: $s^{\prime} \rightarrow \tilde{s}\left(u^{\prime}\right), t^{\prime} \rightarrow \tilde{t}\left(\theta^{\prime}\right)$, and use first-order Taylor expansion to expand $s(u)$ around $s^{\prime}\left(u^{\prime}\right)$ as follows:

$$
s(u)-s\left(u^{\prime}\right) \approx m_{s}\left(u^{\prime}\right)\left(u-u^{\prime}\right)
$$

where

$$
\left.m_{s}\left(u^{\prime}\right) \triangleq \frac{\partial \tilde{s}}{\partial u}(u)\right|_{u=u^{\prime}}=\frac{D_{\mathrm{sd}}}{D_{\mathrm{s} 0} \cos \tilde{\gamma}\left(\tilde{s}\left(u^{\prime}\right)\right)}
$$

Similarily,

$$
\tilde{t}(\theta)-\tilde{t}\left(\theta^{\prime}\right) \approx m_{t}\left(\theta^{\prime}\right)\left(\theta-\theta^{\prime}\right)
$$

where

$$
\left.m_{t}\left(\theta^{\prime}\right) \triangleq \frac{\partial \tilde{t}}{\partial \theta}(\theta)\right|_{\theta=\theta^{\prime}}=\frac{D_{\mathrm{sd}}}{\cos ^{2} \theta^{\prime}} .
$$

Reparameterize variables $s$ and $t$ according to the inversion of cone-to-parallel rebinning as

$$
s \rightarrow \tilde{s}(u), \quad t \rightarrow \tilde{t}(\theta), \quad \beta \rightarrow \tilde{\beta}(\varphi, u), \quad \zeta \rightarrow \tilde{\zeta}(u, v, \theta)
$$

we have the following:

$$
\begin{aligned}
\tilde{\theta}\left(t^{\prime}\right) & =\tilde{\theta}\left(\tilde{t}\left(\theta^{\prime}\right)\right)=\theta^{\prime} \\
\tilde{u}\left(s^{\prime}\right) & =\tilde{u}\left(\tilde{s}\left(u^{\prime}\right)\right)=u^{\prime} \\
\tilde{\varphi}\left(s^{\prime}, \beta\right) & =\varphi+\arcsin \frac{u^{\prime}}{D_{\mathrm{sd}}}-\arcsin \frac{u}{D_{\mathrm{sd}}} \approx \varphi \\
\tilde{\theta}\left(\frac{\Delta_{\mathrm{x}}}{\Delta_{\mathrm{z}}} t^{\prime}\right) & =\tilde{\theta}\left(\frac{\Delta_{\mathrm{x}}}{\Delta_{\mathrm{z}}} \tilde{t}\left(\theta^{\prime}\right)\right)=\arctan \left(\frac{\Delta_{\mathrm{x}}}{\Delta_{\mathrm{z}}} \tan \theta^{\prime}\right) \triangleq \vartheta\left(\theta^{\prime}\right) \\
\tilde{v}(\tilde{s}, \tilde{t}, \zeta) & =\tilde{v}\left(\tilde{s}\left(u^{\prime}\right), \tilde{t}\left(\theta^{\prime}\right), \tilde{\zeta}(u, v, \theta)\right) \approx v-f\left(u^{\prime}, \theta\right)\left(\theta-\theta^{\prime}\right),
\end{aligned}
$$

by exploiting locality approximations $u^{\prime} \approx u, \theta^{\prime} \approx \theta$, where

$$
f\left(u^{\prime}, \theta^{\prime}\right) \triangleq \frac{D_{\mathrm{s} 0} \cos \tilde{\gamma}\left(\tilde{s}\left(u^{\prime}\right)\right)}{\cos \theta^{\prime}}
$$


Plugging (18) and (20) into (16) and changing variables $u^{\prime}=u^{\prime}, v^{\prime}=v-f(u, \theta)\left(\theta-\theta^{\prime}\right)$, the cone-beam system matrix elements can be rebinned and approximated as:

$$
\begin{aligned}
& a(s, t ; \beta, \zeta ; \vec{n}) \approx a_{p}(u, v ; \varphi, \theta ; \vec{n}) \triangleq a(\tilde{s}(u), \tilde{t}(\theta) ; \tilde{\beta}(u, \varphi), \tilde{\zeta}(u, v, \theta) ; \vec{n}) \\
& \approx \iint b\left(m_{s}(u)\left(u-u^{\prime}\right), m_{s, t}(u, \theta)\left(v-v^{\prime}\right)\right) \frac{\Delta_{\mathrm{X}} \Delta_{\mathrm{Z}}}{c(\theta)} \\
& \cdot g\left(\frac{u^{\prime}-u_{\varphi}[\vec{n}]}{\Delta_{\mathrm{X}}}, \frac{v^{\prime}-v_{\varphi, \theta}[\vec{n}]}{c(\theta)} ; \varphi, \vartheta(\theta)\right)\left|m_{s}(u) m_{s, t}(u, \theta)\right| \mathrm{d} u^{\prime} \mathrm{d} v^{\prime}
\end{aligned}
$$

where

$$
m_{s, t}(u, \theta) \triangleq \frac{m_{t}(\theta)}{f(u, \theta)}=\frac{D_{\mathrm{sd}}}{D_{\mathrm{s} 0} \cos \theta^{\prime} \cos \tilde{\gamma}\left(\tilde{s}\left(u^{\prime}\right)\right)}
$$

Consider $\vec{n}$ and $\vec{n}^{\prime}$ values that are sufficiently close to $\vec{n}_{0}$, the location of interest. Let $u_{0}(\varphi) \triangleq u_{\varphi}\left[\vec{n}_{0}\right], v_{0}(\varphi, \theta) \triangleq$ $v_{\varphi, \theta}\left[\vec{n}_{0}\right]$. and $v_{0}(\varphi, \theta) \triangleq v_{\varphi, \theta}\left[\vec{n}_{0}\right]$. Assume $m_{s}(u)$ and $m_{s, t}(u, \theta)$ are fairly smooth over $(u, v)$ :

$$
\begin{aligned}
m_{s}(u) & \approx m_{s}\left(u_{0}(\varphi)\right) \triangleq m_{s, 0}(\varphi) \\
m_{s, t}(u, \theta) & \approx m_{t}\left(u_{0}(\varphi), \theta\right) \triangleq m_{s, t, 0}(\varphi, \theta) .
\end{aligned}
$$

Therefore, we make further approximations using (26) and (27) to (24) as follows:

$$
\begin{aligned}
& a_{p}(u, v ; \varphi, \theta ; \vec{n}) \approx a_{0}\left(u-u_{\varphi}[\vec{n}], v-v_{\varphi, \theta}[\vec{n}] ; \varphi, \theta\right) \\
& \triangleq \iint b_{0}\left(u-u_{\varphi}[\vec{n}]-u^{\prime \prime}, v-v_{\varphi, \theta}[\vec{n}]-v^{\prime \prime} ; \varphi, \theta\right) \\
& \cdot \frac{\Delta_{\mathrm{X}} \Delta_{\mathrm{Z}}}{c(\theta)} g\left(\frac{u^{\prime \prime}}{\Delta_{\mathrm{X}}}, \frac{v^{\prime \prime}}{c(\theta)} ; \varphi, \vartheta(\theta)\right) \mathrm{d} u^{\prime \prime} \mathrm{d} v^{\prime \prime},
\end{aligned}
$$

where

$$
\begin{aligned}
b_{0}(u, v ; \varphi, \theta) & \triangleq\left|m_{s, 0}(\varphi) m_{s, t, 0}(\varphi, \theta)\right| b\left(m_{s, 0}(\varphi) u, m_{s, t, 0}(\varphi, \theta) v\right) \\
a_{0}(u, v ; \varphi, \theta) & \triangleq \iint b_{0}\left(u-u^{\prime}, v-v^{\prime}\right) \frac{\Delta_{\mathrm{X}} \Delta_{\mathrm{z}}}{c(\theta)} g\left(\frac{u^{\prime}}{\Delta_{\mathrm{X}}}, \frac{v^{\prime}}{c(\theta)} ; \varphi, \vartheta(\theta)\right) \mathrm{d} u^{\prime} \mathrm{d} v^{\prime}
\end{aligned}
$$

The second line of (28) makes change of variables $u^{\prime \prime}=u^{\prime}-u_{\varphi}[\vec{n}], v^{\prime \prime}=v^{\prime}-v_{\varphi, \theta}[\vec{n}]$.

Then the elements of the Gram matrix are given exactly by

$$
\begin{aligned}
h_{\mathrm{d}}\left[\vec{n} ; \vec{n}^{\prime}\right] & =\left\{\begin{array}{cc}
{\left[\boldsymbol{A}^{\prime} \boldsymbol{W} \boldsymbol{A}\right]_{j j^{\prime}},} & \vec{n}=\vec{n}_{j} \in \mathcal{S}, \vec{n}^{\prime}=\vec{n}_{j^{\prime}} \in \mathcal{S} \\
0, & \text { otherwise }
\end{array}\right. \\
& =\breve{h}_{\mathrm{d}}\left[\vec{n} ; \vec{n}^{\prime}\right] \eta\left(\vec{x}_{c}[\vec{n}]\right) \eta\left(\vec{x}_{c}\left[\vec{n}^{\prime}\right]\right)
\end{aligned}
$$

where

$$
\breve{h}_{\mathrm{d}}\left[\vec{n} ; \vec{n}^{\prime}\right]=\sum_{k=1}^{n_{s}} \sum_{l=1}^{n_{t}} \sum_{i=1}^{n_{\beta}} \sum_{j=1}^{n_{\zeta}} w\left(s_{k}, t_{\ell} ; \beta_{i}, \zeta_{j}\right) a\left(s_{k}, t_{\ell} ; \beta_{i}, \zeta_{j} ; \vec{n}\right) a\left(s_{k}, t_{\ell} ; \beta_{i}, \zeta_{j} ; \vec{n}^{\prime}\right)
$$

and $\eta\left(\vec{x}_{c}[\vec{n}]\right) \triangleq 1_{\{\vec{n} \in \mathcal{S}\}}, w(s, t ; \beta, \zeta)$ denotes the weighting associated with $\boldsymbol{W}$. We first use integrals to approximate the summations in (32) as follows:

$$
\begin{array}{r}
\breve{h}_{\mathrm{d}}\left[\vec{n} ; \vec{n}^{\prime}\right] \approx \Pi \int_{-\infty}^{\infty} \int_{-\infty}^{\infty} \int_{0}^{2 \pi} \int_{\infty}^{\infty} w(s, t ; \beta, \zeta) \operatorname{rect}\left(\frac{s}{2 s_{\max }}\right) \operatorname{rect}\left(\frac{t}{2 t_{\max }}\right) \operatorname{rect}\left(\frac{\zeta}{2 \zeta_{\max }}\right) \\
\cdot a(s, t ; \beta, \zeta ; \vec{n}) a\left(s, t ; \beta, \zeta ; \vec{n}^{\prime}\right) \mathrm{d} s \mathrm{~d} t \mathrm{~d} \beta \mathrm{d} \zeta,
\end{array}
$$


where $\Pi=1 /\left(\Delta_{\mathrm{S}} \Delta_{t} \Delta_{\beta} \Delta_{\zeta}\right), \Delta_{\mathrm{S}}, \Delta_{t}, \Delta_{\beta}$ and $\Delta_{\zeta}$ are the sampling intervals in $s, t, \beta$ and $\zeta$. Notice that $\breve{h}_{\mathrm{d}}\left[\vec{n} ; \vec{n}^{\prime}\right]$ in (33) is not shift invariant. Make a change of variables from cone-beam coordinates to parallel-beam coordinates, (33) can be rewritten as

$$
\begin{aligned}
\breve{h}_{\mathrm{d}}\left[\vec{n} ; \vec{n}^{\prime}\right] & \approx \Pi \int_{-\frac{\pi}{2}}^{\frac{\pi}{2}} \int_{0}^{2 \pi} \int_{-\infty}^{\infty} \int_{\infty}^{\infty} \bar{w}(u, v ; \varphi, \theta) a_{p}(u, v ; \varphi, \theta ; \vec{n}) a_{p}\left(u, v ; \varphi, \theta ; \vec{n}^{\prime}\right) J(u, \theta) \mathrm{d} u \mathrm{~d} v \mathrm{~d} \varphi \mathrm{d} \theta \\
& \approx \Pi \int_{-\frac{\pi}{2}}^{\frac{\pi}{2}} \int_{0}^{2 \pi} \breve{w}\left(\varphi, \theta ; \vec{n} ; \vec{n}^{\prime}\right) \breve{h}_{\varphi, \theta}\left[\vec{n} ; \vec{n}^{\prime}\right] \mathrm{d} \varphi \mathrm{d} \theta,
\end{aligned}
$$

where $\bar{w}(u, v ; \varphi, \theta)$ is the rebinning weighting as follows:

$$
\bar{w}(u, v ; \varphi, \theta) \triangleq w(s(u), t(\theta) ; \beta(\varphi, u), \zeta(u, v, \theta)) \operatorname{rect}\left(\frac{s(u)}{2 s_{\max }}\right) \operatorname{rect}\left(\frac{t(\theta)}{2 t_{\max }}\right) \operatorname{rect}\left(\frac{\zeta(u, v, \zeta)}{2 \zeta_{\max }}\right),
$$

and for small $\theta, J(u, \theta)$ is the absolute value of the determinant of the Jacobian matrix, given by

$$
J(u, \theta)=\left|\frac{\partial \tilde{s}}{\partial u} \frac{\partial \tilde{t}}{\partial \theta} \frac{\partial \tilde{\beta}}{\partial \varphi} \frac{\partial \tilde{\zeta}}{\partial v}\right|=\left|\frac{D_{\mathrm{sd}}^{2}}{\cos ^{3} \theta \sqrt{D_{\mathrm{s} 0}^{2}-u^{2}}}\right|,
$$

and

$$
\begin{aligned}
\breve{w}\left(\varphi, \theta ; \vec{n} ; \vec{n}^{\prime}\right) & \triangleq \frac{\int_{-\infty}^{\infty} \int_{\infty}^{\infty} \bar{w}(u, v ; \varphi, \theta) a_{p}(u, v ; \varphi, \theta ; \vec{n}) a_{p}\left(u, v ; \varphi, \theta ; \vec{n}^{\prime}\right) J(u, \theta) \mathrm{d} u \mathrm{~d} v}{\int_{-\infty}^{\infty} \int_{\infty}^{\infty} a_{p}(u, v ; \varphi, \theta ; \vec{n}) a_{p}\left(u, v ; \varphi, \theta ; \vec{n}^{\prime}\right) \mathrm{d} u \mathrm{~d} v} \\
\breve{h} & \\
\breve{\varphi}_{\varphi, \theta}\left[\vec{n} ; \vec{n}^{\prime}\right] & \triangleq \int_{-\infty}^{\infty} \int_{\infty}^{\infty} a_{p}(u, v ; \varphi, \theta ; \vec{n}) a_{p}\left(u, v ; \varphi, \theta ; \vec{n}^{\prime}\right) \mathrm{d} u \mathrm{~d} v .
\end{aligned}
$$

To further simply the expression (34), we make further approximations to (37) and (38) based on the fact that $\vec{n}$ and $\vec{n}^{\prime}$ are sufficiently close to $\vec{n}_{0}$ :

$$
\begin{aligned}
\breve{w}\left(\varphi, \theta ; \vec{n} ; \vec{n}^{\prime}\right) & \approx \frac{\int_{-\infty}^{\infty} \int_{\infty}^{\infty} \bar{w}(u, v ; \varphi, \theta) J(u, \theta) a_{0}\left(u-u_{\varphi}[\vec{n}], v-v_{\varphi, \theta}[\vec{n}]\right) a_{0}\left(u-u_{\varphi}[\vec{n}], v-v_{\varphi, \theta}\left[\vec{n}^{\prime}\right]\right) \mathrm{d} u \mathrm{~d} v}{\int_{-\infty}^{\infty} \int_{\infty}^{\infty} a_{0}\left(u-u_{\varphi}[\vec{n}], v-v_{\varphi, \theta}[\vec{n}]\right) a_{0}\left(u-u_{\varphi}\left[\vec{n}^{\prime}\right], v-v_{\varphi, \theta}\left[\vec{n}^{\prime}\right]\right) \mathrm{d} u \mathrm{~d} v} \\
& \approx \frac{\int_{-\infty}^{\infty} \int_{\infty}^{\infty} J_{0}(\varphi, \theta) \bar{w}(u, v ; \varphi, \theta) a_{0}^{2}\left(u-u_{0}(\varphi), v-v_{0}(\varphi, \theta)\right) \mathrm{d} u \mathrm{~d} v}{\int_{-\infty}^{\infty} \int_{\infty}^{\infty} a_{0}^{2}\left(u-u_{0}(\varphi), v-v_{0}(\varphi, \theta)\right) \mathrm{d} u \mathrm{~d} v} \\
& \approx J\left(u_{0}(\varphi), \theta\right) \bar{w}\left(u_{0}(\varphi), v_{0}(\varphi, \theta) ; \varphi, \theta\right) \approx J_{0}(\varphi, \theta) \bar{w}\left(u_{0}(\varphi), v_{0}(\varphi, \theta) ; \varphi, \theta\right) \triangleq w_{0}(\varphi, \theta) \\
\breve{h}_{\varphi, \theta}\left[\vec{n} ; \vec{n}^{\prime}\right] & \approx \int_{-\infty}^{\infty} \int_{\infty}^{\infty} a_{0}\left(u-u_{\varphi}[\vec{n}], v-v_{\varphi, \theta}[\vec{n}]\right) a_{0}\left(u-u_{\varphi}[\vec{n}], v-v_{\varphi, \theta}\left[\vec{n}^{\prime}\right]\right) \mathrm{d} u \mathrm{~d} v \\
& =\int_{-\infty}^{\infty} \int_{\infty}^{\infty} a_{0}(u, v ; \varphi, \theta) a_{0}\left(u+u_{\varphi}[\vec{n}]-u_{\varphi}\left[\vec{n}^{\prime}\right], v+v_{\varphi, \theta}\left[\vec{n}^{\prime}\right]-v_{\varphi, \theta}\left[\vec{n}^{\prime}\right] ; \varphi, \theta\right) \mathrm{d} u \mathrm{~d} v \\
& =\breve{h}_{0}\left(\vec{\Delta} \odot\left(\vec{n}-\vec{n}^{\prime}\right) \cdot \vec{e}_{1}(\vec{\gamma}), \vec{\Delta} \odot\left(\vec{n}-\vec{n}^{\prime}\right) \cdot \vec{e}_{2}(\vec{\gamma}) ; \varphi, \theta\right),
\end{aligned}
$$

where

$$
\begin{aligned}
J_{0}(\varphi, \theta) & \triangleq J\left(u_{0}(\varphi), \theta\right) \\
\breve{h}_{0}(u, v ; \varphi, \theta) & \triangleq a_{0}(u, v ; \varphi, \theta) \star \star a_{0}(u, v ; \varphi, \theta) .
\end{aligned}
$$

Thus, we now have a form that is nearly shift-invariant (except for edge effects).

Combining all the approximations above, we have the expression for the local impulse response:

$$
h_{\mathrm{d}}\left[\vec{n} ; \vec{n}^{\prime}\right] \approx \Pi \int_{-\frac{\pi}{2}}^{\frac{\pi}{2}} \int_{0}^{2 \pi} w_{0}(\varphi, \theta) h_{\varphi, \theta}\left[\vec{n} ; \vec{n}^{\prime}\right] \mathrm{d} \varphi \mathrm{d} \theta,
$$


where

$$
h_{\varphi, \theta}\left[\vec{n} ; \vec{n}^{\prime}\right] \triangleq \breve{h}_{0}\left(\vec{\Delta} \odot\left(\vec{n}-\vec{n}^{\prime}\right) \cdot \vec{e}_{1}, \vec{\Delta} \odot\left(\vec{n}-\vec{n}^{\prime}\right) \cdot \vec{e}_{2} ; \varphi, \theta\right) \eta\left(\vec{x}_{c}[\vec{n}]\right) \eta\left(\vec{x}_{c}\left[\vec{n}^{\prime}\right]\right) .
$$

As shown in 2D fan-beam case, ${ }^{5}$ the edge effects in (31) are a main concern in accurate variance prediction. Here we use a similar approach to take the edge effects into account to find the local frequency response. As in 2D fan-beam case, we refer all displacements relative to the point $\vec{n}_{0}$ as follows:

$$
h_{\varphi, \theta}\left[\vec{n} ; \vec{n}^{\prime}\right] \approx h_{\varphi, \theta}\left[\vec{n}_{0}+\vec{n} ; \vec{n}_{0}\right]=\breve{h}_{0}\left(\vec{\Delta} \odot \vec{n} \cdot \vec{e}_{1}, \vec{\Delta} \odot \vec{n} \cdot \vec{e}_{2} ; \varphi, \theta\right) \eta_{2}\left(\vec{x}_{c}[\vec{n}]\right) \triangleq \tilde{h}_{\varphi, \theta}\left[\vec{n} ; \vec{n}_{0}\right],
$$

where $\eta_{2}(\vec{x}) \triangleq \eta\left(\vec{x}_{0}+\vec{x}\right) \eta\left(\vec{x}_{0}\right)$, and then approximate $\eta_{2}(\vec{x})$ as following:

$$
\eta_{2}(\vec{x}) \approx \eta_{0}(\vec{x})=\eta(\vec{x}) \eta\left(\vec{x}_{0}\right) .
$$

This choice also yields a local impulse response that is symmetric in $\vec{n}$ provided $\eta(\vec{x})$ is symmetric itself. We focus on a symmetric $\eta(\vec{x})$, such as elliptical cylinder or sphere hereafter. The final form of local impulse response follows from (43), (45) and (46):

$$
h_{\mathrm{d}}\left[\vec{n} ; \vec{n}^{\prime}\right] \approx \Pi \int_{-\frac{\pi}{2}}^{\frac{\pi}{2}} \int_{0}^{2 \pi} w_{0}(\varphi, \theta) \tilde{h}_{\varphi, \theta}\left[\vec{n} ; \vec{n}_{0}\right] \mathrm{d} \varphi \mathrm{d} \theta .
$$

\subsection{Local Frequency Response of Gram Matrix}

To find the DSFT $H_{\mathrm{d} 0}(\vec{\omega})$ of $h_{\mathrm{d}}\left[\vec{n} ; \vec{n}^{\prime}\right]$, we need $H_{\varphi, \theta}(\vec{\omega})$ first, the spectrum of $\tilde{h}_{\varphi, \theta}\left[\vec{n} ; \vec{n}_{0}\right]$, because by linearity of DSFT, taking the DSFT of (47) yields the following result:

$$
H_{\mathrm{d} 0}(\vec{\omega})=\Pi \int_{-\frac{\pi}{2}}^{\frac{\pi}{2}} \int_{0}^{2 \pi} w_{0}(\varphi, \theta) H_{\varphi, \theta}(\vec{\omega}) \mathrm{d} \varphi \mathrm{d} \theta .
$$

Define a "tube like" function $s_{\varphi, \theta}(\vec{x}) \triangleq \breve{h}_{0}\left(\vec{x} \cdot \vec{e}_{1}, \vec{x} \cdot \vec{e}_{2} ; \varphi, \theta\right) \eta_{0}(\vec{x})$, then we have $\tilde{h}_{\varphi, \theta}\left[\vec{n} ; \vec{n}_{0}\right]=s_{\varphi, \theta}(\vec{\Delta} \odot \vec{n})$. The spectrum of $\tilde{h}_{\varphi, \theta}\left[\vec{n} ; \vec{n}_{0}\right]$ can be found as follows:

$$
\begin{aligned}
H_{\varphi, \theta}(\vec{\omega}) & =\sum_{\vec{n}} \tilde{h}_{\varphi, \theta}\left[\vec{n} ; \vec{n}_{0}\right] \mathrm{e}^{-\imath(\vec{\omega} \cdot \vec{n})}=\sum_{\vec{n}} s_{\varphi, \theta}(\vec{\Delta} \odot \vec{n}) \mathrm{e}^{-\imath(\vec{\omega} \cdot \vec{n})} \\
& \approx \frac{1}{\Delta_{\mathrm{X}}^{2} \Delta_{\mathrm{Z}}} \iiint s_{\varphi, \theta}(\vec{x}) \mathrm{e}^{-\imath \frac{1}{\vec{\Delta}} \odot(\vec{\omega} \cdot \vec{x})} \mathrm{d} \vec{x}=\frac{1}{\Delta_{\mathrm{X}}^{2} \Delta_{\mathrm{Z}}} S_{\varphi, \theta}\left(\frac{1}{2 \pi \vec{\Delta}} \odot \vec{\omega}\right),
\end{aligned}
$$

where $s_{\varphi, \theta}(\vec{x}) \stackrel{2 \mathrm{D} \mathrm{FT}}{\longleftrightarrow} S_{\varphi, \theta}(\vec{\nu})$.

To preserve the non-negative definiteness of the Gram Matrix, we choose

$$
\eta_{0}(\vec{x})=\operatorname{tri}\left(\frac{\vec{x} \cdot \vec{e}_{3}}{d_{0}(\varphi, \vartheta(\theta))}\right)
$$

where $d_{0}(\varphi, \vartheta(\theta))$ is the intersecting length of the profile passing though $\vec{n}_{0}$ and the finite support at direction $(\varphi+$ $\pi / 2, \vartheta(\theta))$. Therefore the local frequency response $H_{0}(\varrho, \Phi, \Theta)$ of the step-and-shoot cone-beam Gram matrix around $\vec{n}_{0}$ is

$$
H_{0}(\varrho, \Phi, \Theta) \approx \Upsilon \int_{-\theta_{\max }}^{\theta_{\max }} \int_{0}^{2 \pi} w_{0}(\varphi, \theta) S_{\varphi, \theta}(\varrho, \Phi, \Theta) \mathrm{d} \varphi \mathrm{d} \theta
$$

where the constant $\Upsilon \triangleq \frac{\Pi}{\Delta_{\mathrm{X}}^{2} \Delta_{\mathrm{Z}}}$. The 3D FT of $s_{\varphi, \theta}(\vec{x})$ has the following form according to the rotation property:

$$
S_{\varphi, \theta}(\vec{\nu})=\left|A_{0}\left(\vec{\nu} \cdot \vec{e}_{1}(\vec{\gamma}), \vec{\nu} \cdot \vec{e}_{2}(\vec{\gamma}) ; \varphi, \theta\right)\right|^{2} d_{0}(\varphi, \vartheta(\theta)) \operatorname{sinc}^{2}\left(d_{0}(\varphi, \vartheta(\theta)) \vec{\nu} \cdot \vec{e}_{3}(\vec{\gamma})\right),
$$

where $A_{0}\left(\nu_{u}, \nu_{v} ; \varphi, \theta\right)$ is the 2D FT of $a_{0}(u, v ; \varphi, \theta)$ with respect to $(u, v)$, including the effects of basis function and detector blur:

$$
A_{0}\left(\nu_{u}, \nu_{v} ; \varphi, \theta\right)=B_{0}\left(\nu_{u}, \nu_{v} ; \varphi, \theta\right) G\left(\nu_{u}, \nu_{v} ; \varphi, \theta\right)
$$


where $B_{0}\left(\nu_{u}, \nu_{v} ; \varphi, \theta\right) G\left(\nu_{u}, \nu_{v} ; \varphi, \theta\right)$ are the 2D FT of $b_{0}(u, v ; \varphi, \theta)$ and $\frac{\Delta_{\mathrm{X}} \Delta_{\mathrm{Z}}}{c(\theta)} p\left(\frac{u}{\Delta_{\mathrm{X}}}, \frac{v}{c(\theta)} ; \varphi, \vartheta(\theta)\right)$ with respect to $(u, v)$, respectively.

As $\theta \rightarrow 0$ and $d_{0}(\varphi, \vartheta(\theta)) \rightarrow \infty$, one can show that for large $\varrho$, the $\operatorname{sinc}^{2}$ term is sharply peaked at near $\varphi=\Phi \pm \pi$ :

$$
d_{0}(\varphi, \vartheta(\theta)) \operatorname{sinc}^{2}\left(d_{0}(\varphi, \vartheta(\theta)) \vec{\nu} \cdot \vec{e}_{3}(\vec{\gamma})\right) \rightarrow \delta\left(\vec{\nu} \cdot \vec{e}_{3}(\vec{\gamma})\right)=\delta(\varrho \sin (\varphi-\Phi) \cos \Theta)
$$

Therefore we consider the following approximations to (51):

$$
H_{0}(\varrho, \Phi, \Theta) \approx \Upsilon \int_{-\theta_{\max }}^{\theta_{\max }} w_{0}(\Phi, \theta) \int_{0}^{2 \pi} S_{\varphi, \theta}(\varrho, \Phi, \Theta) \mathrm{d} \varphi \mathrm{d} \theta
$$

where $S_{\varphi, \theta}(\varrho, \Phi, \Theta) \triangleq S_{\varphi, \theta}\left(\varrho \vec{e}_{\Phi, \Theta}\right)$. If we consider "ideal" cubic basis function $\chi(\vec{x})=\operatorname{rect}_{3}(\vec{x})$, then

$$
\begin{aligned}
A_{0}\left(\nu_{u}, \nu_{v} ; \varphi, \theta\right)=\Delta_{\mathrm{X}}^{2} \Delta_{\mathrm{Z}} & \operatorname{sinc}\left(\Delta_{\mathrm{X}} \nu_{u} \cos \varphi-c(\theta) \nu_{v} \sin \varphi \sin \vartheta(\theta)\right) \\
& \cdot \operatorname{sinc}\left(\Delta_{\mathrm{X}} \nu_{u} \sin \varphi+c(\theta) \nu_{v} \cos \varphi \sin \vartheta(\theta)\right) \\
& \cdot \operatorname{sinc}\left(c(\theta) \nu_{v} \cos \vartheta(\theta)\right) \operatorname{sinc}\left(\frac{\Delta_{\mathrm{S}} \nu_{u}}{m_{s, 0}(\varphi)}\right) \operatorname{sinc}\left(\frac{\Delta_{t} \nu_{v}}{m_{s, t, 0}(\varphi, \theta)}\right) .
\end{aligned}
$$

\section{3D QUADRATIC REGULARIZATION}

For a discrete-space $3 \mathrm{D}$ object $\mu[\vec{n}]$, a typical quadratic roughness penalty is given as

$$
R(\boldsymbol{\mu})=\sum_{\vec{n}} \sum_{l=1}^{L} r_{l, 0} \frac{1}{2}\left(\left(c_{l} * * * \mu\right)[\vec{n}]\right)^{2},
$$

where $r_{l, 0}$ values are design parameters that affect the directionality of the regularization and hence the shape of the PSF. Each $c_{l}[\vec{n}]$ is a (typically) high-pass filter. For a first-order difference: $c_{l}[\vec{n}]=\xi_{l}\left(\delta_{3}[\vec{n}]-\delta_{3}\left[\vec{n}-\vec{m}_{l}\right]\right.$ ), where $\xi_{l}=\left\|\vec{m}_{l}\right\|^{-v / 2}, \vec{m}_{l}$ denotes the spatial offsets to the neighboring voxels, and $v$ is the power of weights for diagonal neighbors that can be chosen by the user. For example, common practice chooses $v=1 .^{7,8}$

Applying Parseval's theorem, the local frequency response of $\boldsymbol{R}$ for a $\Lambda$-order (where $\Lambda \in \mathbb{N}$ ) difference can be found in a similar form of 2D fan-beam case as follows:

$$
R_{0}(\varrho, \Phi, \Theta)=\sum_{l=1}^{L} r_{l, 0} \xi_{l}^{2 \Lambda} 4 \sin ^{2 \Lambda}\left(\pi \varrho\left(\vec{\Delta} \odot \vec{e}_{\Phi, \Theta}\right) \cdot \vec{m}_{l}\right) .
$$

In 3D geometry, $L=13$ for the second order neighborhood.

\section{SIMULATION RESULTS}

We first used a smaller image and sinogram to validate the local frequency responses $H_{0}(\varrho, \Phi, \Theta)$ and $R_{0}(\varrho, \Phi, \Theta)$ for a given location. We simulated a step-and shoot cone-beam CT system with an cylindrical 2D dectector with quarter detector offset. The corresponding sinogram size was 111 samples in $s$, spaced by $\Delta_{\mathrm{S}} \approx 8 \mathrm{~mm}, 32$ samples in $t$, spaced by $\Delta_{t} \approx 8.8$ $\mathrm{mm}, 20$ samples in $\zeta$, spaced by $\Delta_{\zeta} \approx 5 \mathrm{~mm}$, and 123 source angular positions over $360^{\circ}$ for each $\zeta$. The image is of size $64 \times 62 \times 20$ and the object is an ellipsoid. The voxel size is of $7.8 \mathrm{~mm}, 7.8 \mathrm{~mm}$ and $5 \mathrm{~mm}$. An cylindrical support is used with radius $r_{s}=164.1 \mathrm{~mm}$ and height $80 \mathrm{~mm}$. In this case, $d_{0}(\varphi, \vartheta(\theta))=r_{s} / \cos \vartheta(\theta)$.

In our simulation, we used the distance-driven (DD) projectors developed by Deman et al. The 3D forward projector works by mapping voxel boundaries and detector boundaries onto a common plane specifying by $\left(r_{\varphi}, z\right)$, where $r_{\varphi} \triangleq \max (|\cos \varphi|,|\sin \varphi|)$. This method approximates the total contribution of voxel $j$ to detector $k$ by the following expression: ${ }^{6}$

$$
P_{j k} \approx \frac{\Delta}{\cos \beta \cos \theta} \frac{o_{1}}{\Delta_{\mathrm{S}}} \frac{o_{2}}{\Delta_{t}} \mu_{k}
$$



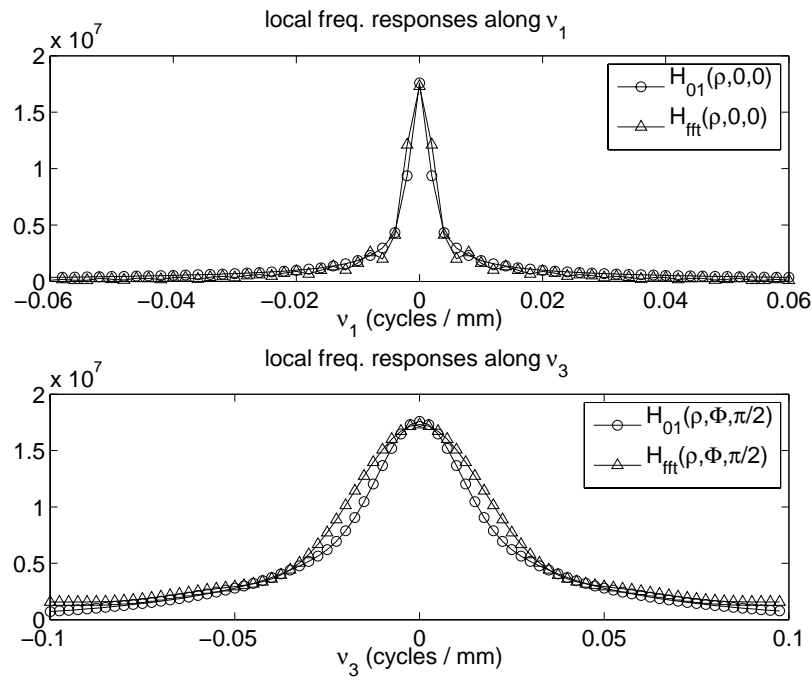

Figure 1. Profiles of local frequency responses of Gram matrix at image center.
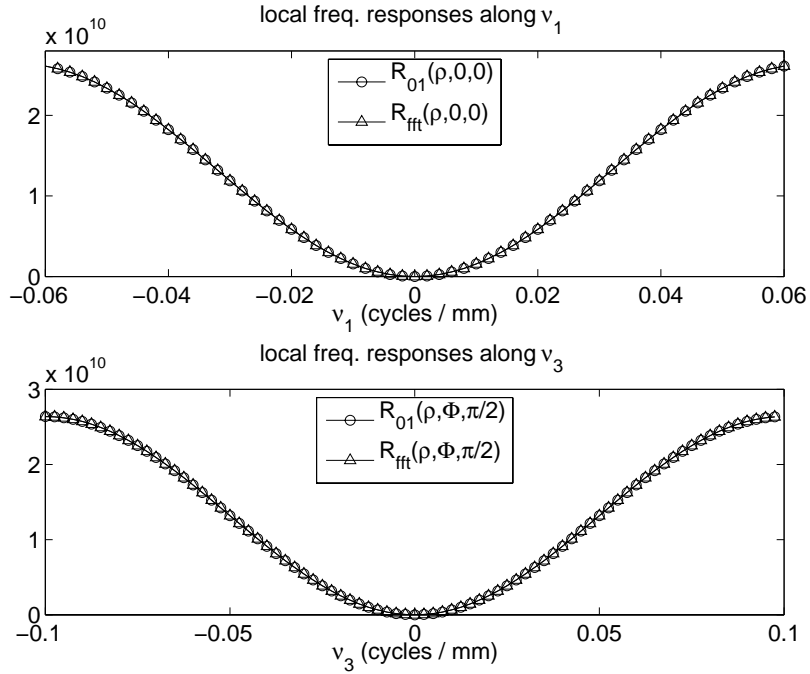

Figure 2. Profiles of local frequency responses of quadratic regularization at image center.

where $\Delta$ is isotropic voxel size and $o_{1}, o_{2}$ are the overlapped length between the mapped boundaries of voxel $j$ and detector $k$ onto $r_{\varphi}$-axis and z-axis, respectively.

Therefore, for the DD projector, instead of (56), $A\left(\nu_{u}, \nu_{v} ; \varphi, \theta\right)$ is given by

$$
A\left(\nu_{u}, \nu_{v} ; \varphi, \theta\right)=\Delta_{\mathrm{X}}^{2} \Delta_{\mathrm{Z}} \operatorname{sinc}\left(\min \left(\frac{\Delta_{\mathrm{S}}}{m_{s, 0}(\varphi)}, \Delta_{\mathrm{X}} \max (|\cos \varphi|,|\sin \varphi|)\right) \nu_{u}\right) \operatorname{sinc}\left(\min \left(\frac{\Delta_{t}}{m_{s, t, 0}(\varphi, \theta)}, \Delta_{\mathrm{z}}\right) \nu_{v}\right) .
$$

- Quadratically Penalized Unweighted Likelihood (QPUL) case:

We first investigated the unweighted case where $\vec{n}_{0}$ is at image center. Fig. 1 and Fig. 2 show the $\nu_{1}$-axis and $\nu_{3}$ axis profiles of local frequency responses $H_{0}(\varrho, \Phi, \Theta)$ and $R_{0}(\varrho, \Phi, \Theta)$. The normalized root-mean-squared (NRMS) difference between the standard deviations predicted by DFT method (2) and AVP methods (5) with (55) and (57) is less than $1 \%$. 


\section{ACKNOWLEDGMENTS}

This project is supported in part by NIH/NCI grant 1P01 CA87634 and by GE Healthcare.

\section{REFERENCES}

1. J. A. Fessler, "Mean and variance of implicitly defined biased estimators (such as penalized maximum likelihood): Applications to tomography," IEEE Trans. Im. Proc. 5, pp. 493-506, Mar. 1996.

2. J. Qi and R. H. Huesman, "Theoretical study of lesion detectability of MAP reconstruction using computer observers," IEEE Trans. Med. Imag. 20, pp. 815-22, Aug. 2001.

3. J. W. Stayman and J. A. Fessler, "Efficient calculation of resolution and covariance for fully-3D SPECT," IEEE Trans. Med. Imag. 23, pp. 1543-56, Dec. 2004.

4. Y. Zhang, J. A. Fessler, and J. Hsieh, "Fast variance image predictions for quadratically regularized statistical image reconstruction in fan-beam tomography," in Proc. IEEE Nuc. Sci. Symp. Med. Im. Conf., 4, pp. 1929-33, 2005.

5. Y. Zhang-O'Connor and J. A. Fessler, "Fast predictions of variance images for fan-beam transmission tomography with quadratic regularization," IEEE Trans. Med. Imag. , 2006. To appear.

6. B. De Man and S. Basu, "Distance-driven projection and backprojection in three dimensions," Phys. Med. Biol. 49, pp. 2463-75, June 2004.

7. J. A. Fessler, "Penalized weighted least-squares image reconstruction for positron emission tomography," IEEE Trans. Med. Imag. 13, pp. 290-300, June 1994.

8. J. Nuyts and J. A. Fessler, "A penalized-likelihood image reconstruction method for emission tomography, compared to post-smoothed maximum-likelihood with matched spatial resolution," IEEE Trans. Med. Imag. 22, pp. 1042-52, Sept. 2003. 